\title{
Diffusion-weighted magnetic resonance imaging in bladder cancer: comparison of readout-segmented and single-shot EPI techniques
}

\author{
Haihu Chen ${ }^{1 \dagger}$, Luguang Chen ${ }^{1 \dagger}$, Fang Liu', Jianping Lu', Chuanliang $X \mathrm{U}^{2^{*}}$ and Li Wang ${ }^{1 *}$
}

\begin{abstract}
Background: To evaluate whether readout-segment echo-planar imaging (RS-EPI) can provide better image quality in assessing bladder cancer than single-shot echo-planar imaging (SS-EPI) and to compare quantitative imaging parameters derived from both techniques.

Methods: Seventy patients with bladder lesions were enrolled and underwent diffusion-weighted imaging on a 3 Tesla magnetic resonance scanner using axial RS-EPI and SS-EPI techniques. Two observers independently assessed the susceptibility, detectability, motion artefacts and blurring of the images using qualitative scores. The signal-tonoise ratio (SNR), signal intensity ratio (SIR), contrast-to-noise ratio (CNR) and ADC values of the bladder lesions were measured and compared between the two techniques and between two observers. Qualitative and quantitative comparisons of image quality were performed using the Wilcoxon signed-rank test and paired t-test. In addition, the agreement of the ADC measurements was evaluated using ICC values and Bland-Altman plots.
\end{abstract}

Results: Sixty-eight patients were included in the final analysis. The scores of image susceptibility, detectability and blurring for RS-EPI were significantly higher than those for SS-EPI (all $p<0.05$ ), while the motion artefact was not. There were significant differences between RS-EPI and SS-EPI in the CNR and SIR values (all $p<0.05$ ) but not in the SNR or ADC values (all $p>0.05$ ). The ICC values and Bland-Altman plots also showed excellent agreement between the measured ADC values of the bladder lesions.

Conclusions: The RS-EPI technique provides significantly better image quality in patients with bladder cancer than the SS-EPI technique, without a significant difference in the ADC value.

Keywords: Urinary bladder neoplasms, Diffusion, Echo-planar imaging, Magnetic resonance imaging

\section{Background}

Bladder cancer is one of the most common malignant tumours in urology. According to the statistics, there were 429,800 new cases of bladder cancer and 165,100 related deaths worldwide in 2012 [1]. In 2015, there were approximately 80,000 new cases of bladder cancer and 34,000 related deaths in China [2]. The treatment and prognosis of bladder cancer mainly depend on the pathological stage

\footnotetext{
*Correspondence: xuchuanliang@vip.126.com; wangli_changhai@163.com ${ }^{+}$Haihu Chen and Luguang Chen contributed equally to this work. ${ }^{2}$ Department of Urology, Changhai Hospital of Shanghai, The Second Military Medical University, No.168 Changhai Road, Shanghai 200433, China 'Department of Radiology, Changhai Hospital of Shanghai, The Second Military Medical University, No.168 Changhai Road, Shanghai 200433, China
}

and grade of bladder cancer. Magnetic resonance imaging (MRI) has been used to evaluate bladder lesions, with merits such as non-ionizing radiation, good image contrast, multi-parameter imaging and high spatial resolution [3]. T1-weighted imaging has advantages in the diagnosis of fat infiltration around bladder cancer; however, the bladder wall shows hypointensity similar to that of bladder tumours on T1-weighted imaging, and the value of this method for evaluating whether the tumour has infiltrated the muscle layer is limited $[4,5]$. T2-weighted imaging has good soft tissue resolution and can clearly show the relationship between bladder tumours and muscles, but it is difficult to use for distinguishing tumors from benign

(C) The Author(s). 2019 Open Access This article is distributed under the terms of the Creative Commons Attribution 4.0 International License (http://creativecommons.org/licenses/by/4.0/), which permits unrestricted use, distribution, and 
lesions, such as inflammation, fibrosis and oedema, leading to excessive staging $[6,7]$. Dynamic contrast-enhanced MRI can be used to differentiate bladder tumours and muscles according to the different enhancement modes of the lesions, while the enhancement of small blood vessels located at the base of the tumour is similar to that of the tumour, which also leads to over-staging [8, 9]. Diffusionweighted imaging (DWI) is a functional imaging technique that reflects the state of tissues by detecting the motion of water molecules in vivo. Bladder tumours show high signals on DWI, while benign changes, such as inflammation, fibrosis and oedema, caused by tumour growth show low signals, and the bladder wall shows slightly higher signals; therefore, DWI can be used to avoid excessive staging caused by the inability to distinguish inflammatory lesions, fibrotic changes, oedema and small vessels $[6,10,11]$. In recent years, several studies have reported that DWI plays a significant role in preoperative staging and grading for the diagnosis of bladder cancer [6, 11-14].

Single-shot echo-planar imaging (SS-EPI) is a commonly used method due to its relative insensitivity to motion-induced phase errors and short acquisition time [15]. However, SS-EPI images are characterized by blurring along the phase encoding direction due to $\mathrm{T} 2$ * decay and are sensitive to off-resonance effects [16]. Susceptibility variation at tissue-air interfaces can result in magnetic susceptibility artefacts, especially at higher field strengths. In addition, it is usually difficult to use diffusion-weighted images with low spatial resolution that are acquired using the SS-EPI technique to assess small lesions. In recent years, a new multi-shot technique, referred to as readout-segmented echo-planar imaging (RS-EPI), has been proposed for improved the spatial resolution and decreased susceptibility-induced image distortion and $\mathrm{T} 2 *$ blurring [16]. This new multishot EPI technique is based on the segmentation of $\mathrm{k}$ space into several partitions in the readout-encoding direction at each excitation, which allows for a reduction in the echo spacing and shortens the time required for the k-space trajectory in the phase-encoding direction. In addition, a two-dimensional navigator echo was integrated into the RS-EPI technique and used for phase correction between different shots, making the method more robust with respect to motion-induced phase errors. A number of reports have shown improved image quality using the RS-EPI technique in various tissues in vivo, such as the paediatric brain, paranasal sinus, orbit, cholesteatoma, liver, breast, kidney, prostate and rectum [17-25]. However, few studies have reported the assessment of bladder lesions using RS-EPI. We hypothesized that the RS-EPI could be a promising method for DWI in bladder cancer.

Therefore, the aims of the present study were to evaluate whether RS-EPI can provide better image quality in assessing bladder cancer than SS-EPI and to compare quantitative imaging parameters derived from RS-EPI and SS-EPI.

\section{Methods \\ Patients}

Between June 2017 and December 2017, 70 consecutively patients suspected of bladder cancer were recruited in this retrospective study. The inclusion criteria were as follows: (1) bladder cancer confirmed by preoperative cystoscopy; (2) surgical treatment performed within 2 weeks after MRI examination; and (3) postoperative pathological staging. Patients with no histological evidence of bladder cancer and magnetic resonance imaging contraindications were excluded from this study. This study was approved by the local institutional review board, and written informed consent was waived for each patient in this retrospective study.

\section{Magnetic resonance imaging}

All patients were examined using on a 3 Tesla MRI scanner (Skyra, Siemens medical solution, Germany) with a standard 18-channel phased-array body coil and an integrated spine coil. Each patient fasted for $4 \mathrm{~h}$ and was instructed to drink $500 \mathrm{ml}$ of water to dilate the bladder before the MRI examination. In addition, patients were given regular breathing training to reduce respiratory artefacts. The imaging protocols included axial T1weighted turbo spin echo (TSE), T2-weighted TSE, axial DWI with RS-EPI and SS-EPI and contrast-enhanced

Table 1 The main parameters of MRI protocols

\begin{tabular}{|c|c|c|c|c|c|c|c|c|c|c|c|}
\hline Protocols & $\mathrm{TR} / \mathrm{TE}$ (ms) & $\mathrm{FOV}\left(\mathrm{mm}^{2}\right)$ & Matrix & Thickness (mm) & Gap (mm) & Slices & $\begin{array}{l}\text { Acceleration } \\
\text { factor }\end{array}$ & $\begin{array}{l}\text { b-values } \\
\left(\mathrm{s} / \mathrm{mm}^{2}\right)\end{array}$ & Averages & $\begin{array}{l}\text { Readout } \\
\text { segments }\end{array}$ & Scan time \\
\hline T1W TSE & $680 / 20$ & $220 \times 220$ & $180 \times 256$ & 4 & 0.8 & 30 & 3 & - & 2 & - & $2^{\prime} 2^{\prime \prime}$ \\
\hline T2W TSE & $7500 / 101$ & $200 \times 200$ & $320 \times 320$ & 4 & 0.4 & 20 & 2 & - & 2 & - & $3^{\prime} 9^{\prime \prime}$ \\
\hline SS-EPI & $3000 / 66$ & $176 \times 220$ & $72 \times 90$ & 4 & 0 & 16 & 2 & 0,1000 & 1,12 & 1 & $2^{\prime}$ \\
\hline RS-EPI & $3400 / 63$ & $156 \times 260$ & $102 \times 170$ & 4 & 0 & 16 & 2 & 0,1000 & 1,4 & 5 & $4^{\prime} 35^{\prime \prime}$ \\
\hline T1W VIBE & $3.33 / 1.23$ & $234 \times 360$ & $154 \times 192$ & 3 & 0 & 30 & 4 & - & 1 & - & $16^{\prime \prime}$ \\
\hline
\end{tabular}


Table 2 Criteria for qualitative comparison of image quality in diffusion-weighted imaging in patients with bladder cancer

\begin{tabular}{l} 
Susceptibility \\
1: marked distortion was seen \\
3: little distortion was seen \\
4: no distortion was seen \\
Detectability \\
1: not visible \\
2: partial visibility \\
3: visibility \\
4: excellent visibility \\
Motion artifact \\
1: marked artifact \\
2: moderate artifact \\
3: slight artifact \\
4: no artifact \\
Image blurring \\
1: the bladder wall definition was not visible \\
2: the bladder wall definition was partially visible \\
3: the bladder wall definition was visible \\
4: the bladder wall definition was clearly visible \\
\hline
\end{tabular}

three-dimensional T1-weighted volumetric interpolated breath-hold examination (3D T1W VIBE). The main parameters of these protocols are presented in Table 1.

\section{Image analysis}

All images were sent to an advanced workstation (Leonardo, Siemens medical solution, Germany) for further analysis. Apparent diffusion coefficient (ADC) maps of the RS-EPI and SS-EPI images were reconstructed online in the scanner using the monoexponential mode. The RS-EPI and SS-EPI DWI images were evaluated by two independent observers (H.C and F.L., with 4 and 5 years of experience in pelvic MRI, respectively) in terms of the susceptibility, detectability, motion artefact and blurring of the lesion images using a quantitative 4-point scale (Table 2).

Freehand regions of interest (ROIs) were manually outlined to profile the lesion on the ADC maps of the RS-EPI and SS-EPI images and then copied them to the corresponding diffusion-weighted images, with b-value $=1000 \mathrm{~s} /$ $\mathrm{mm}^{2}$. ROIs were drawn on the diffusion-weighted images with reference to non-contrast and contrast-enhanced T1W images to avoid the inclusion of hemorrhagic and necrotic tissues. ROIs of normal tissue were drawn in the gluteus maximus. The mean value and standard deviation of each ROI were recorded (Fig. 1). The signal-to-noise ratio (SNR) was calculated as the ratio between the mean signal amplitude of the lesion $\left(\mathrm{SI}_{\text {lesion }}\right)$ in the diffusionweighted images and the standard deviation of the background noise $\left(\sigma_{\text {noise }}\right)$. The signal intensity ratio (SIR) was defined as the ratio of the $\mathrm{SI}_{\text {lesion }}$ to the mean signal intensity of normal tissue $\left(\mathrm{SI}_{\text {normal }}\right)$. The contrast-to-noise ratio (CNR) was determined as the difference between the $\mathrm{SI}_{\text {lesion }}$ and $\mathrm{SI}_{\text {normal }}$ divided by the standard deviation of the lesion $\left(\sigma_{\text {lesion }}\right)$ and normal tissue $\left(\sigma_{\text {normal }}\right)$. The SNR, SIR and CNR were calculated using the following formulas:

$$
\begin{aligned}
& \mathrm{SNR}=S I_{\text {lesion }} / \sigma_{\text {noise }} \\
& \mathrm{SIR}=S I_{\text {lesion }} / S I_{\text {normal }} \\
& \mathrm{CNR}=\left|S I_{\text {lesion }}-S I_{\text {normal }}\right| / \sqrt{\sigma_{\text {lesion }^{2}+\sigma_{\text {normal }}{ }^{2}}}
\end{aligned}
$$

\section{Statistical analysis}

Statistical analyses were carried out using SPSS (version 16.0, SPSS Inc., Chicago, IL, USA) and MedCalc (version 13.0.0.0, MedCalc Software, Mariakerke, Belgium) software. The Kolmogorov-Smirnov test was used to assess the normality of the distribution of continuous variables. Quantitative data are described as the mean \pm standard
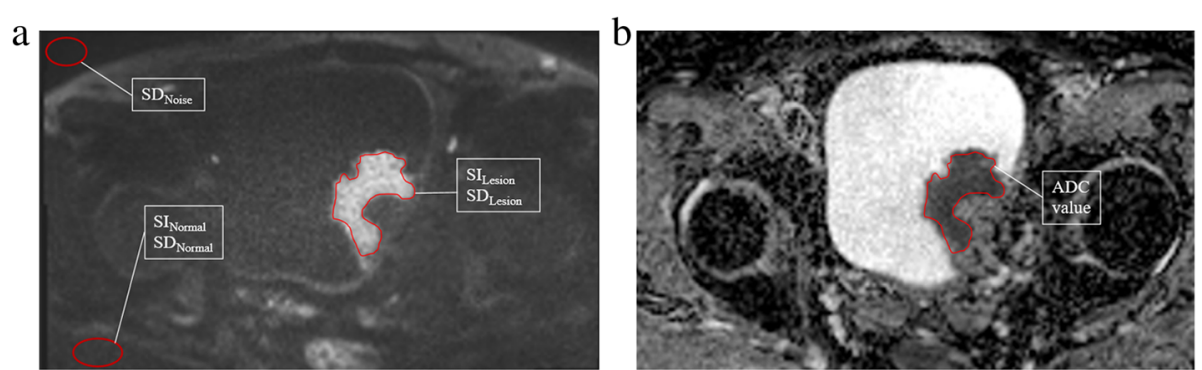

Fig. 1 Representative images of outlined ROIs in bladder lesions. a DWl: b-value $=1000 \mathrm{~s} / \mathrm{mm}^{2}$. ROls were delineated to determine the signal intensity and standard deviation of the lesion and normal reference tissues, as was the standard deviation of the noise level. b ROI outlined for estimating the ADC value of the bladder lesion 
Table 3 Qualitative comparison of image scores between RS-EPI and SS-EPI techniques in patients with bladder cancer

\begin{tabular}{llllll}
\hline Observer & Protocol & Susceptibility & Detectability & Motion artifact & Image blurring \\
\hline Observer 1 & RS-EPI & $3.50 \pm 0.56$ & $3.94 \pm 0.24$ & $3.68 \pm 0.50$ & $3.71 \pm 0.46$ \\
& SS-EPI & $3.22 \pm 0.84$ & $3.82 \pm 0.42$ & $3.63 \pm 0.54$ & $2.74 \pm 0.44$ \\
& p-value & 0.000 & 0.005 & 3.083 & 0.000 \\
Observer 2 & RS-EPI & $3.41 \pm 0.67$ & $3.82 \pm 0.42$ & $3.69 \pm 0.47$ & $3.76 \pm 0.43$ \\
& SS-EPI & $3.22 \pm 0.81$ & 0.011 & 0.317 & $3.01 \pm 0.47$ \\
& p-value & 0.000 & 0.000 & 0.000 \\
\hline
\end{tabular}

Note: RS-EPI Readout segmented echo-planar imaging, SS-EPI Single-shot echo-planar imaging

deviation. Differences in image quality between RS-EPI and SS-EPI were assessed using the Wilcoxon signed-rank test. Significant differences between the two techniques and between the two observers in the quantitative parameters (SNR, SIR and CNR) of image quality and the ADC values were assessed by using paired t-test. Agreement between the two techniques and between the two observers for the $\mathrm{ADC}$ values were evaluated using the intraclass correlation coefficient (ICC) and Bland-Altman plots $[26,27]$. ICC values $<0.4$ indicated poor agreement; 0.40.75 , good agreement; and $>0.75$, excellent agreement. In addition, the mean percent difference and 95\% limits of agreement between the paired ADC measurements were calculated. A $p$-value less than 0.05 was regarded as statistically significant.

\section{Results}

\section{Patients}

A total of 70 patients were recruited for RS-EPI and SSEPI DWI. However, 1 patient was excluded because of the lack of histological evidence of bladder cancer, and 1 patient was excluded due to contraindications to magnetic resonance imaging. Therefore, 68 patients $(60$ males and 8 females, mean age: $66.1 \pm 14.7$ years) completed all MRI examinations. Histology showed that 49 patients had high-grade urothelial carcinoma and 19 patients had low-grade urothelial carcinoma. Pathological staging showed that 48 patients had T1, 2 patients had T2, 5 patients had T3a, 4 patients had T3b, 3 patients had T4, in addition, 6 patients were identified as having muscle invasive bladder cancer (> T1), but the exact stages were not determined because the whole bladder was not resected and pathological sections of the bladder tumour and the bladder wall could not be obtained. The tumour size ranged from $5 \mathrm{~mm}$ to $65 \mathrm{~mm}$ (mean: $26.6 \pm 13.2 \mathrm{~mm}$ ).

\section{Qualitative comparison of image quality}

The results of the image quality analysis for the RS-EPI and SS-EPI techniques are shown in Table 3. There were significant differences between the two techniques in the susceptibility, detectability and blurring of the images of the bladder lesions (all $p<0.05$ ) but not the motion artefacts $(3.68 \pm 0.50$ vs. $3.63 \pm 0.54$ and $3.69 \pm 0.47$ vs. $3.66 \pm 0.54, p=0.083$ and 0.317 ) for both observers. Representative images from patients with low- and highgrade urothelial carcinoma are shown in Figs. 2 and 3. Figure 4 shows dot plots of image quality for the RS-EPI
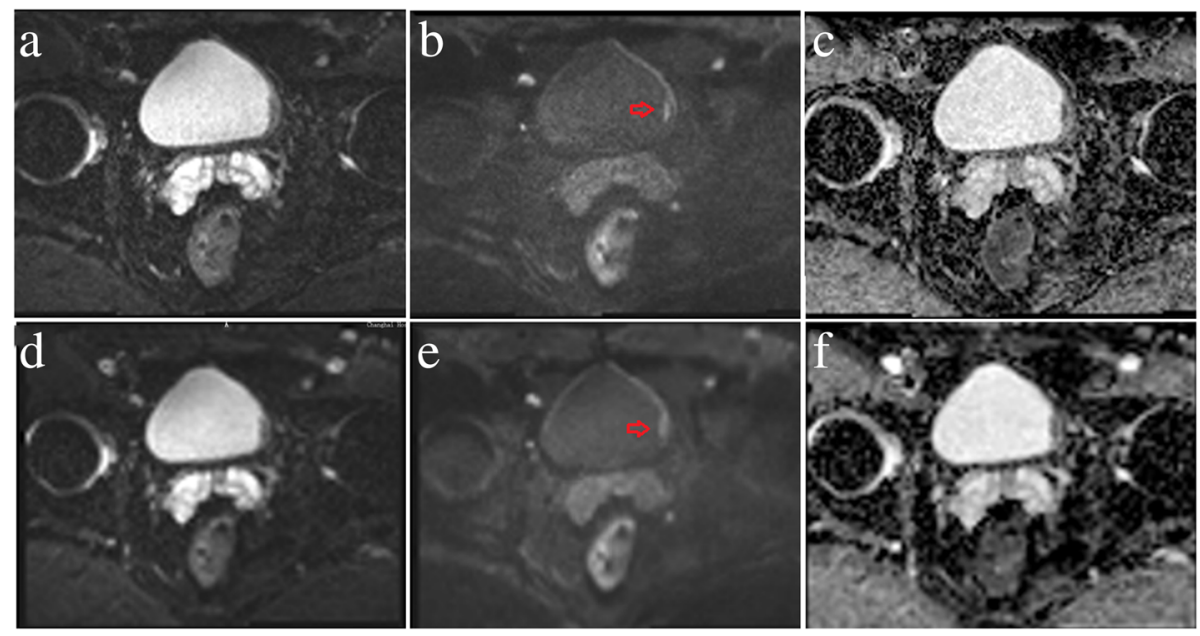

Fig. 2 A 39-year-old male with low-grade urothelial carcinoma in the left wall of the bladder (red arrows). RS-EPI: b-value $=0 \mathrm{~s} / \mathrm{mm}^{2}(\mathbf{a}) ; \mathrm{b}-\mathrm{value}=$ $1000 \mathrm{~s} / \mathrm{mm}^{2}$ (b); ADC map (c). SS-EPI: b-value $=0 \mathrm{~s} / \mathrm{mm}^{2}$ (d); b-value $=1000 \mathrm{~s} / \mathrm{mm}^{2}$ (e); ADC map (f) 

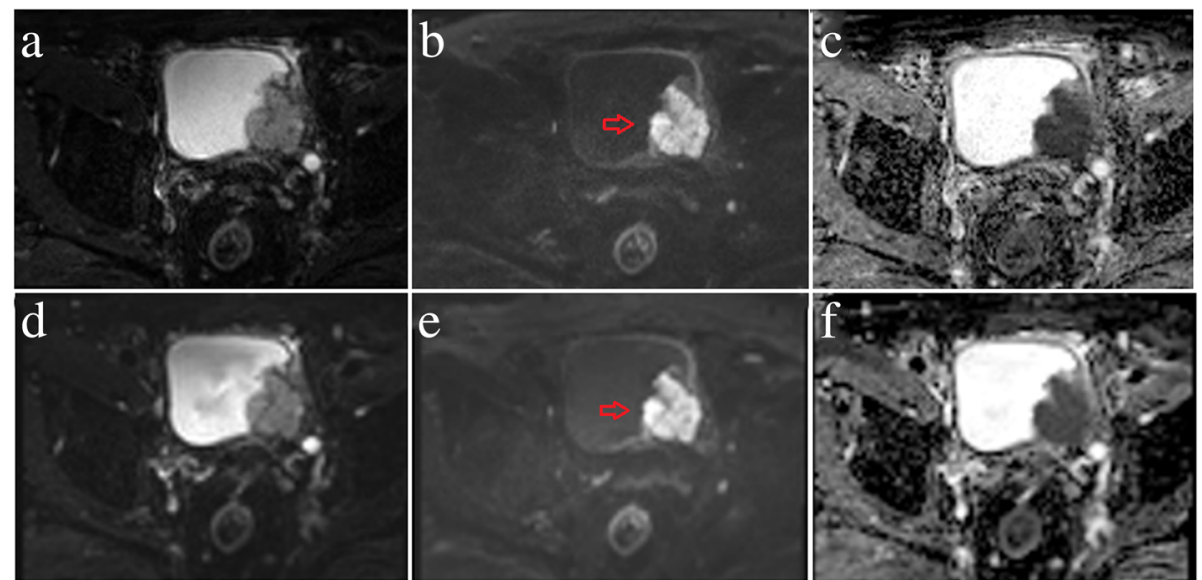

Fig. 3 A 70-year-old male with high-grade urothelial carcinoma in the left posterior wall of the bladder (red arrows). RS-EPI: b-value $=0 \mathrm{~s} / \mathrm{mm}^{2}(\mathbf{a})$; b-value $=1000 \mathrm{~s} / \mathrm{mm}^{2}$ (b); ADC map (c). SS-EPI: b-value $=0 \mathrm{~s} / \mathrm{mm}^{2}$ (d); b-value $=1000 \mathrm{~s} / \mathrm{mm}^{2}$ (e); ADC map (f)

and SS-EPI techniques. The mean image quality scores were higher for RS-EPI than SS-EPI.

\section{Quantitative comparison of image quality parameters}

A quantitative comparison of image quality parameters between RS-EPI and SS-EPI for the two observers is shown in Table 4 . There were significant differences between the RS-EPI and SS-EPI techniques in the CNR and SIR values (all $p<0.05$ ), while no significant difference was observed in the SNR value $(p>0.05)$. Table 5 shows comparisons of the SNR, SIR and CNR measurements between Observer 1 and Observer 2 for both techniques; no significant differences were observed in those measurements (all $p>0.05$ ).

\section{Quantitative comparison of $A D C$ values}

A quantitative comparison and the agreement of the ADC values are shown in Table 6. There were no
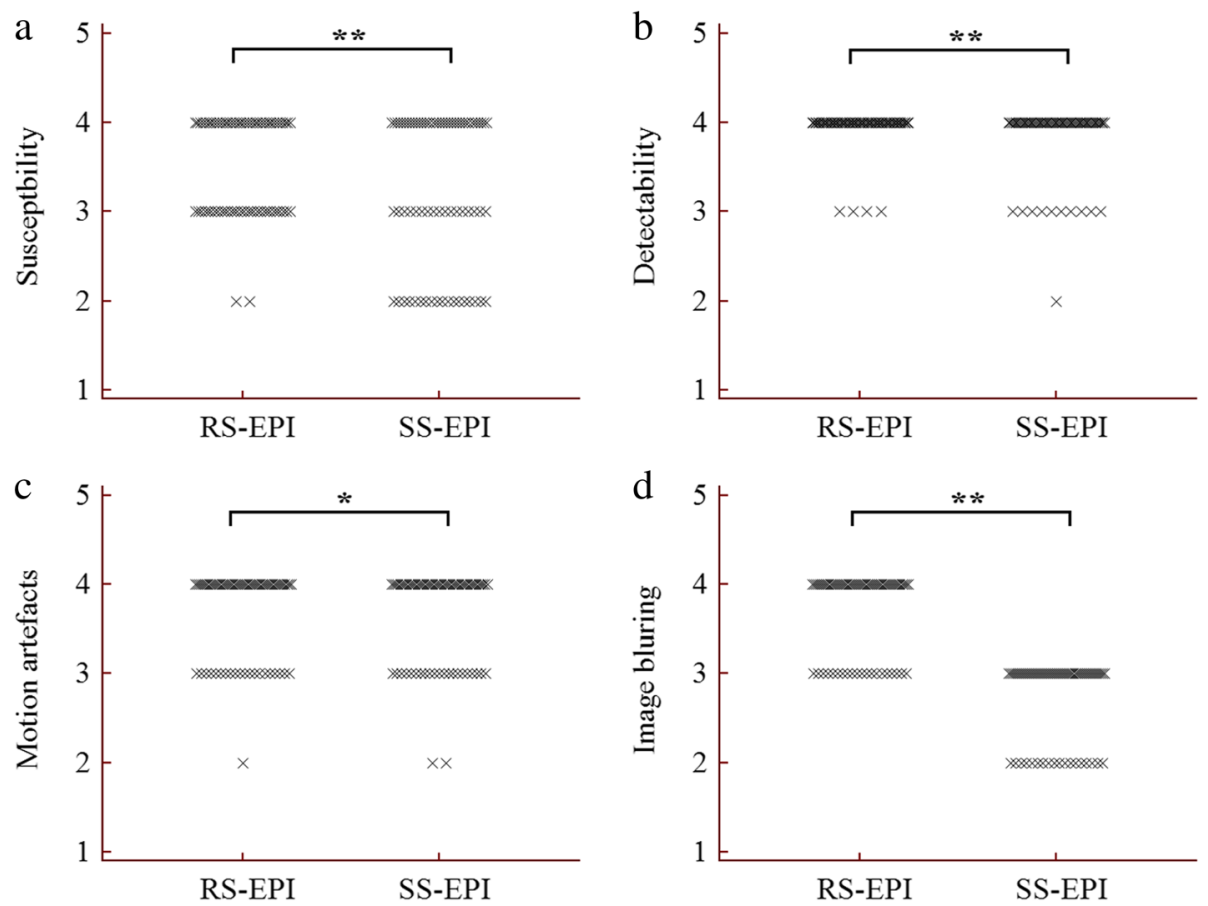

Fig. 4 Qualitative comparison of image scores between RS-EPI and SS-EPI in patients with bladder cancer. a Scores for susceptibility; b Scores for detectability; c Scores for motion artefact; d Scores for image blurring 
Table 4 Quantitative comparison of image quality between RS-EPI and SS-EPI techniques in patients with bladder cancer

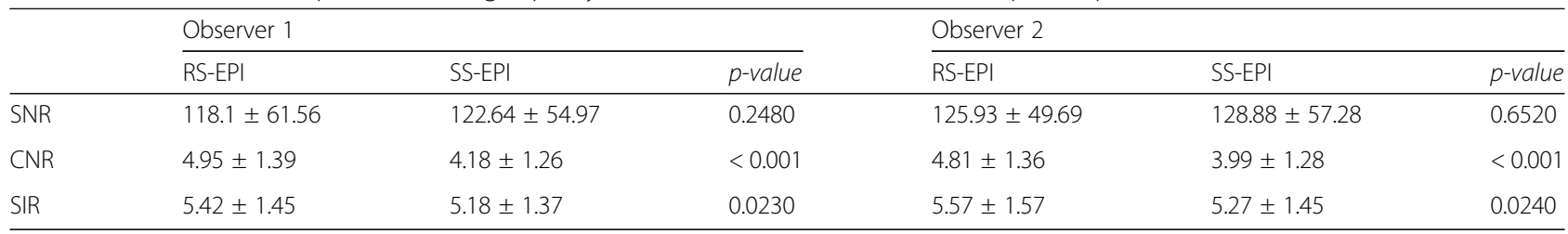

Note: SNR Signal-to-noise ratio, CNR Contrast-to-noise ratio, SIR Signal intensity ratio

significant differences between the two techniques $(1.170 \pm$ 0.215 vs. $1.179 \pm 0.221 \times 10^{-3} \mathrm{~mm}^{2} / \mathrm{s}, p=0.322$ for Observer 1 , and $1.174 \pm 0.207$ vs. $1.179 \pm 0.226 \times 10^{-3} \mathrm{~mm}^{2} / \mathrm{s}$, $p=0.604$ for Observer 2, respectively) for either observer, in addition, there were no significant differences between the two observers $(1.170 \pm 0.215$ vs. $1.174 \pm$ $0.207 \times 10^{-3} \mathrm{~mm}^{2} / \mathrm{s}, p=0.302$ for RS-EPI, and $1.179 \pm$ 0.221 vs. $1.179 \pm 0.226 \times 10^{-3} \mathrm{~mm}^{2} / \mathrm{s}, p=0.956$ for SS-EPI, respectively) obtained using RS-EPI and SS-EPI. In addition, the agreement of the ADC values between the two technique and between the two observers was excellent, with ICC values ranging from 0.937 to 0.990 . Moreover, the mean percent difference and limits of agreement between Observer 1 and Observer $2\left(\mathrm{O}_{1}\right.$ and $\mathrm{O}_{2}$ ) for the ADC values obtained using RS-EPI (ADC $\mathrm{RS}$ ) and SS-EPI $\left(\mathrm{ADC}_{\mathrm{SS}}\right)$ were $-0.6(-7.3-6.2)$ and 0.1 (5.6-5.7), respectively (Fig. 5a, b). The mean percent difference and limits of agreement between the two techniques for the ADC values measured by $\mathrm{O}_{1}$ and $\mathrm{O}_{2}$ were -0.7 (-13.2-11.7) and -0.1 (-12.5-12.3), respectively (Fig. 5c, d).

\section{Discussion}

In the present study, we demonstrated significantly higher image quality and better image contrast in bladder DWI using RS-EPI than SS-EPI. In addition, no significant difference was observed in the SNR or ADC value, which shows that RS-EPI is a promising method for reducing image artefacts and improving image quality in bladder cancer. The blurring and detectability of bladder cancer lesion was significantly improved with RS-EPI. The RSEPI technique provides significantly better image quality than SS-EPI at 3 Tesla and can potentially provide better image quality in patients with bladder cancer.

DWI is a useful MRI technique and has been used to characterize the pathophysiology of lesions in clinical practice; it offers functional and structural information on in vivo tissues without ionizing radiation or contrast administration [28]. Several studies have reported that DWI plays a significant role in routine MRI examinations and serves as a good supplement to improve the accuracy of assessing the properties and biological behaviours of bladder cancer [6, 29-31]. In addition, ADC values, which are derived from diffusion-weighted images, have been shown to have potential as imaging biomarkers for predicting the histopathological grade and indicating the aggressiveness of bladder cancer [11, 12, 14, 32, 33]. However, routine DWI is based on the single-shot EPI technique, which is sensitive to susceptibility and distortion. In the present study, a new multi-shot EPI technique (i.e., RSEPI) was used and qualitatively and quantitatively compared in terms of image quality with SS-EPI. The qualitative assessment showed that RS-EPI is superior to SS-EPI in susceptibility, image blurring and lesion detectability. This difference may be explained by the fact that the segmented trajectory through $\mathrm{k}$-space along the readout-encoding direction decreased the echo spacing and accelerated traversal of the $\mathrm{k}$-space along the phase-encoding and readout directions [16]. Our findings are similar to previously reported results $[18,19,22,34-36]$. Motion artefacts in RS-EPI images were not significantly different from those in SS-EPI images. On the one hand, while SS-EPI is insensitive to motion artefacts, a two-dimensional navigator echo was integrated into the RS-EPI technique and used to correct data corrupted because of shot-to-shot phase errors in DWI, rendering RS-EPI immune to those artefacts [16]. On the other hand, the bladder is located in the pelvic cavity and is less affected by respiratory motion artefacts. Therefore, improved image quality was obtained with the RS-EPI technique.

The CNR of bladder lesions in RS-EPI images was significantly better than that lesions in SS-EPI images, and

Table 5 Quantitative comparison of image quality between the two observers in patients with bladder cancer

\begin{tabular}{|c|c|c|c|c|c|c|}
\hline & \multicolumn{3}{|l|}{ RS-EPI } & \multicolumn{3}{|l|}{ SS-EPI } \\
\hline & Observer 1 & Observer 2 & $p$-value & Observer 1 & Observer 2 & $p$-value \\
\hline$\overline{S N R}$ & $118.1 \pm 61.56$ & $125.93 \pm 49.69$ & 0.150 & $122.64 \pm 54.97$ & $128.88 \pm 57.28$ & 0.310 \\
\hline CNR & $4.95 \pm 1.39$ & $4.81 \pm 1.36$ & 0.236 & $4.18 \pm 1.26$ & $3.99 \pm 1.28$ & 0.080 \\
\hline SIR & $5.42 \pm 1.45$ & $5.57 \pm 1.57$ & 0.083 & $5.18 \pm 1.37$ & $5.27 \pm 1.45$ & 0.368 \\
\hline
\end{tabular}

Note: SNR Signal-to-noise ratio, CNR Contrast-to-noise ratio, SIR Signal intensity ratio 
Table 6 Quantitative comparison and agreement of ADC values in patients with bladder cancer

\begin{tabular}{llllll}
\hline & RS-EPI & SS-EPI & $p$-value & ICC & $95 \% \mathrm{Cl}$ \\
\hline Observer 1 & $1.170 \pm 0.215^{\mathrm{a}}$ & $1.179 \pm 0.221^{\mathrm{a}}$ & 0.322 & 0.937 & $0.900-0.961$ \\
Observer 2 & $1.174 \pm 0.207^{\mathrm{a}}$ & $1.179 \pm 0.226^{\mathrm{a}}$ & 0.604 & 0.940 & $0.904-0.962$ \\
p-value & 0.302 & 0.956 & $/$ & $/$ & $/$ \\
ICC & 0.984 & 0.990 & $/$ & $/$ & $/$ \\
$95 \% \mathrm{Cl}$ & $0.974-0.990$ & $0.984-0.994$ & $/$ & $/$ & $/$ \\
\hline
\end{tabular}

Note: ICC intraclass coefficient, $\mathrm{Cl}$ Confidence interval

a, $A D C$ values, $\times 10^{-3} \mathrm{~mm}^{2} / \mathrm{s}$

our results are consistent with those of previous studies $[20,22,25,37]$. Better visualization of bladder lesions can be achieved using the RS-EPI technique. However, several studies have reported no significant difference in the CNR between the two techniques $[17,18,36]$. There was a significant difference in the SIR of bladder lesions between RS-EPI and SS-EPI in the present study. The gluteus maximus was used as a reference for calculating the normalized signal intensity of the bladder lesions so that the SIR values could be compared among the patients.

The SNR of bladder lesions in RS-EPI images was similar to those of lesions in SS-EPI images, and no significant difference was observed between the techniques in this study. Our results are consistent with those of Byeon et al., who compared RS-EPI and SS-EPI in the diagnosis of acute infarction of the brain stem and posterior fossa [18]. Several studies found that the SNR of RS-EPI images was higher than that of SS-EPI images [20, 22, 24, 25, 35], while other reports showed lower SNR values for RS-EPI images [17, 19, 36-39]. The inconsistent results of those studies could be explained by the fact that the SNR is a relative value and depends on the exact imaging parameters being used, such as the magnetic field strength, minimum TE, FOV, matrix, signal average, number of segments, echo spacing, gradient performance, combination of b-values and $\mathrm{T} 2$ value for the tissue. In the present study, the minimum TE, inplane resolution, number of segments and signal average were $63 \mathrm{~ms}, 1.53 \times 1.53 \mathrm{~mm}^{2}, 5$ and 4 for RS-EPI and $66 \mathrm{~ms}, 2.44 \times 2.44 \mathrm{~mm}^{2}, 1$ and 12 for SS-EPI, respectively, whereas the other parameters were kept consistent. Therefore, while a similar SNR was obtained using both techniques, the RS-EPI technique provided highresolution diffusion-weighted images that contributed to the detection of small lesions (Fig. 2).

The mean ADC values of bladder lesions obtained using RS-EPI and SS-EPI were not significantly different and are in good agreement with previously reported data

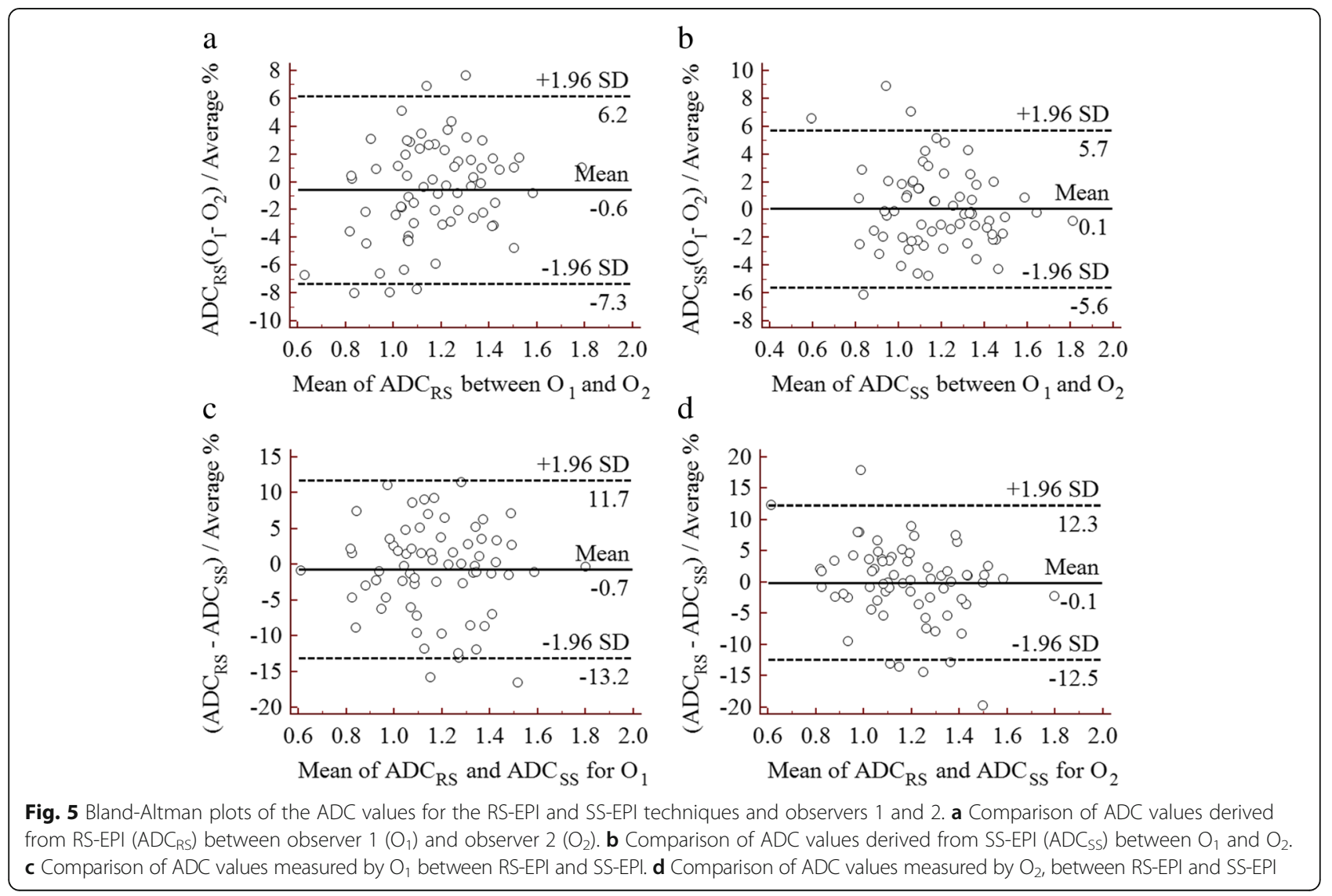


$[17,18,20,25,35,40]$. Nevertheless, Zhao et al. found that the ADC values of sinonasal lesions on RS-EPI were lower than those on SS-EPI [36]. Moreover, $\mathrm{Xu}$ et al. reported that RS-EPI DWI produced significantly lower ADC values than did SS-EPI DWI in patients with orbital tumours [37]. The disagreement between the two methods in the ADC may be explained by the fact that different imaging parameters, such as the magnetic field strength, b-value, TE, spatial resolution, diffusion direction and imaging technology vendor, were used in those studies. In this study, there was excellent reproducibility between the two methods and between the two observers in the measured ADC values of the bladder lesions, as indicated by the ICC values. Moreover, the Bland-Altman plots show the excellent reproducibility of those measurements and narrow intervals of agreement compared with the average values obtained in evaluating the ADC values of bladder lesions (Fig. 5). The improved image quality of RS-EPI and equivalent ADC values derived using RS-EPI and SS-EPI suggest that we may adopt the new method as a substitute for the conventional EPI technique for imaging bladder lesions.

The present study has several limitations. First, compared with SS-EPI, the scan time for imaging bladder lesions using RS-EPI was relatively long (RS-EPI vs SS-EPI: $4 \mathrm{~min}$ $35 \mathrm{~s}$ vs. $2 \mathrm{~min}$ ), and new techniques for accelerating the scan (e.g., simultaneous multi-slice imaging) and optimizing the imaging parameters would overcome this concern. Second, no comparison of the diagnostic efficiency of SSEPI and RS-EPI in differentiating between high- and lowgrade urothelial carcinoma was performed, and because of the relatively small sample size of patients with low-grade urothelial carcinoma, we will recruit more patients for evaluation in future studies. Third, the delineation of ROIs in the bladder lesions was based on a single-slice method in which the maximum lesion size was outlined. However, the effects of ROI methods were not compared in this study, but we will perform such comparisons in future work.

\section{Conclusions}

In conclusion, the RS-EPI technique provides significantly improved image quality in patients with bladder cancer compared with the SS-EPI technique, without a significant difference in the ADC value. The RS-EPI DWI technique may serve as a promising tool for evaluating patients with bladder lesions in clinical practice.

\footnotetext{
Abbreviations

ADC: Apparent diffusion coefficient; CNR: Contrast-to-noise ratio; DWI: Diffusion-weighted imaging; FOV: Field of view; ICC: Intraclass correlation coefficient; MRI: Magnetic resonance imaging; ROI: Region of interest; RS-EPI: Readout-segmented echo-planar imaging; SIR: Signal intensity ratio; SNR: Signal-to-noise ratio; SS-EPI: Single-shot echo-planar imaging; TA: Acquisition time; TR/TE: Repetition time/echo time; TSE: Turbo spin echo; VIBE: Volumetric interpolated breath-hold examination
}

\section{Acknowledgements}

N/A.

\section{Authors' contribution}

LW, JL and CX conceived of the present idea. LW, LC and CX designed the study. Data acquisition was performed by HC. LC performed the statistical analysis. $\mathrm{LC}, \mathrm{HC}$, and $\mathrm{FL}$ contributed to the data analysis and interpretation. $\mathrm{LC}$ and $\mathrm{HC}$ were major contributors and contributed equally to writing the manuscript. All the authors read and approved the final manuscript.

\section{Funding}

This work was supported by the National Key Clinical Specialist Construction Programs of China (Grant Number N/A), the Technology Innovation Action Project of Science and Technology Commission of Shanghai City (16411969700), the Project of Excellent Academic Leader of Science and Technology Committee of Shanghai City (17XD1404900), and the Program of Leading Talent of Shanghai City (N/A).

\section{Availability of data and materials}

The datasets used and/or analyzed during the current study are available from the corresponding author on reasonable request.

Ethics approval and consent to participate

This study was approved by our institutional ethics committee.

\section{Consent for publication}

Written informed consent was waived from each patient due to this retrospective study.

\section{Competing interests}

The authors declare that they have no competing interests.

Received: 8 October 2018 Accepted: 5 August 2019

Published online: 27 August 2019

\section{References}

1. Torre LA, Bray F, Siegel RL, Ferlay J, Lortet-Tieulent J, Jemal A. Global cancer statistics, 2012. CA Cancer J Clin. 2015:65:87-108.

2. Chen W, Zheng R, Baade PD, Zhang S, Zeng H, Bray F, Jemal A, Yu XQ, He J. Cancer statistics in China, 2015. CA Cancer J Clin. 2016;66:115-32.

3. Lee $\mathrm{CH}$, Tan $\mathrm{CH}$, Faria SC, Kundra V. Role of imaging in the local staging of urothelial carcinoma of the bladder. AJR Am J Roentgenol. 2017;208:1193-205.

4. de Haas RJ, Steyvers MJ, Futterer JJ. Multiparametric MRI of the bladder: ready for clinical routine? AJR Am J Roentgenol. 2014;202:1187-95.

5. $\mathrm{Ng}$ CS. Radiologic diagnosis and staging of renal and bladder cancer. Semin Roentgenol. 2006:41:121-38.

6. Takeuchi M, Sasaki S, Ito M, Okada S, Takahashi S, Kawai T, Suzuki K, Oshima H, Hara M, Shibamoto Y. Urinary bladder cancer: diffusion-weighted MR imaging--accuracy for diagnosing $T$ stage and estimating histologic grade. Radiology. 2009;251:112-21.

7. Verma S, Rajesh A, Prasad SR, Gaitonde K, Lall CG, Mouraviev V, Aeron G, Bracken RB, Sandrasegaran K. Urinary bladder cancer: role of MR imaging. Radiographics. 2012;32:371-87.

8. Wang HJ, Pui MH, Guo Y, Li SR, Guan J, Zhang XL, Cai HS. Multiparametric 3-T MRI for differentiating low-versus high-grade and category T1 versus T2 bladder urothelial carcinoma. AJR Am J Roentgenol. 2015;204:330-4.

9. Gupta N, Sureka B, Kumar MM, Malik A, Bhushan TB, Mohanty NK. Comparison of dynamic contrast-enhanced and diffusion weighted magnetic resonance image in staging and grading of carcinoma bladder with histopathological correlation. Urol Ann. 2015;7:199-204.

10. Yamada Y, Kobayashi S, Isoshima S, Arima K, Sakuma H, Sugimura Y. The usefulness of diffusion-weighted magnetic resonance imaging in bladder cancer staging and functional analysis. J Cancer Res Ther. 2014;10:878-82.

11. Takeuchi M, Sasaki S, Naiki T, Kawai N, Kohri K, Hara M, Shibamoto Y. MR imaging of urinary bladder cancer for T-staging: a review and a pictorial essay of diffusion-weighted imaging. J Magn Reson Imaging. 2013;38:1299-309.

12. Wu LM, Chen XX, Xu JR, Zhang XF, Suo ST, Yao OY, Fan Y, Hu J. Clinical value of T2-weighted imaging combined with diffusion-weighted imaging in preoperative T staging of urinary bladder cancer: a large-scale, multiobserver prospective study on 3.0-T MRI. Acad Radiol. 2013;20:939-46. 
13. Ohgiya Y, Suyama J, Sai S, Kawahara M, Takeyama N, Ohike N, Sasamori H, Munechika J, Saiki M, Onoda Y, et al. Preoperative T staging of urinary bladder Cancer: efficacy of stalk detection and diagnostic performance of diffusion-weighted imaging at 3T. Magn Reson Med Sci. 2014;13:175-81.

14. Yoshida S, Koga F, Masuda H, Fujii Y, Kihara K. Role of diffusion-weighted magnetic resonance imaging as an imaging biomarker of urothelial carcinoma. Int J Urol. 2014;21:1190-200.

15. Turner R, Bihan DL. Single-shot diffusion imaging at 2.0 tesla. J Magn Reson. 1990;86:445-52

16. Porter DA, Heidemann RM. High resolution diffusion-weighted imaging using readout-segmented echo-planar imaging, parallel imaging and a twodimensional navigator-based reacquisition. Magn Reson Med. 2009;62:468-75.

17. Bogner W, Pinker-Domenig K, Bickel H, Chmelik M, Weber M, Helbich TH, Trattnig S, Gruber S. Readout-segmented echo-planar imaging improves the diagnostic performance of diffusion-weighted MR breast examinations at 3 . O T. Radiology. 2012;263:64-76.

18. Byeon J, Kim JY, Cho AH. Readout-segmented echo-planar imaging in diffusion-weighted MR imaging of acute infarction of the brainstem and posterior fossa: comparison of single-shot echo-planar diffusion-weighted sequences. Clin Imaging. 2015;39:765-9.

19. Holdsworth SJ, Yeom K, Skare S, Gentles AJ, Barnes PD, Bammer R. Clinical application of readout-segmented- echo-planar imaging for diffusionweighted imaging in pediatric brain. AJNR Am J Neuroradiol. 2011:32:1274-9.

20. Kim YJ, Kim SH, Kang BJ, Park CS, Kim HS, Son YH, Porter DA, Song BJ. Readout-segmented echo-planar imaging in diffusion-weighted $\mathrm{mr}$ imaging in breast cancer: comparison with single-shot echo-planar imaging in image quality. Korean J Radiol. 2014;15:403-10.

21. Li L, Wang L, Deng M, Liu H, Cai J, Sah VK, Liu J. Feasibility study of 3-T DWI of the prostate: readout-segmented versus single-shot Echo-planar imaging. AJR Am J Roentgenol. 2015;205:70-6.

22. Morelli J, Porter D, Ai F, Gerdes C, Saettele M, Feiweier T, Padua A, Dix J, Marra M, Rangaswamy R, et al. Clinical evaluation of single-shot and readout-segmented diffusion-weighted imaging in stroke patients at $3 \mathrm{~T}$. Acta Radiol. 2013;54:299-306.

23. Tokoro H, Fujinaga Y, Ohya A, Ueda K, Shiobara A, Kitou Y, Ueda H, Kadoya M. Usefulness of free-breathing readout-segmented echo-planar imaging (RESOLVE) for detection of malignant liver tumors: comparison with singleshot echo-planar imaging (SS-EPI). Eur J Radiol. 2014;83:1728-33.

24. Wu CJ, Wang Q, Zhang J, Wang XN, Liu XS, Zhang YD, Shi HB. Readoutsegmented echo-planar imaging in diffusion-weighted imaging of the kidney: comparison with single-shot echo-planar imaging in image quality. Abdom Radiol (NY). 2016:41:100-8.

25. Xia CC, Liu X, Peng WL, Li L, Zhang JG, Meng WJ, Deng XB, Zuo PL, Li ZL. Readout-segmented echo-planar imaging improves the image quality of diffusion-weighted MR imaging in rectal cancer: comparison with single-shot echo-planar diffusion-weighted sequences. Eur J Radiol. 2016;85:1818-23.

26. Shrout PE, Fleiss JL. Intraclass correlations: uses in assessing rater reliability. Psychol Bull. 1979;86:420-8.

27. Bland JM, Altman DG. Statistical methods for assessing agreement between two methods of clinical measurement. Lancet. 1986;1:307-10.

28. Lin WC, Chen JH. Pitfalls and limitations of diffusion-weighted magnetic resonance imaging in the diagnosis of urinary bladder Cancer. Transl Oncol. 2015;8:217-30

29. El-Assmy A, Abou-El-Ghar ME, Mosbah A, El-Nahas AR, Refaie HF, Hekal IA, El-Diasty $\mathrm{T}$, Ibrahiem el $\mathrm{H}$. Bladder tumour staging: comparison of diffusionand T2-weighted MR imaging. Eur Radiol. 2009;19:1575-81.

30. Thoeny HC, Forstner R, De Keyzer F. Genitourinary applications of diffusionweighted MR imaging in the pelvis. Radiology. 2012;263:326-42.

31. Avcu S, Koseoglu MN, Ceylan K, Bulut MD, Unal O. The value of diffusionweighted MRI in the diagnosis of malignant and benign urinary bladder lesions. Br J Radiol. 2011:84:875-82.

32. Kobayashi S, Koga F, Kajino K, Yoshita S, Ishii C, Tanaka H, Saito K, Masuda H, Fujii $Y$, Yamada T, et al. Apparent diffusion coefficient value reflects invasive and proliferative potential of bladder cancer. J Magn Reson Imaging. 2014; 39:172-8.

33. Sevcenco S, Ponhold L, Heinz-Peer G, Fajkovic H, Haitel A, Susani M, Shariat SF, Szarvas T, Baltzer PA. Prospective evaluation of diffusion-weighted MRI of the bladder as a biomarker for prediction of bladder cancer aggressiveness. Urol Oncol. 2014;32:1166-71.
34. Algin O, Aydin H, Ozmen E, Ocakoglu G, Bercin S, Porter DA, Kutluhan A. Detection of cholesteatoma: high-resolution DWI using RS-EPI and parallel imaging at 3 tesla. J Neuroradiol. 2017:44:388-94.

35. Thian $\mathrm{YL}$, Xie W, Porter DA, Weileng AB. Readout-segmented echo-planar imaging for diffusion-weighted imaging in the pelvis at 3T-A feasibility study. Acad Radiol. 2014;21:531-7.

36. Zhao M, Liu Z, Sha Y, Wang S, Ye X, Pan Y, Wang S. Readout-segmented echo-planar imaging in the evaluation of sinonasal lesions: a comprehensive comparison of image quality in single-shot echo-planar imaging. Magn Reson Imaging. 2016;34:166-72.

37. Xu X, Wang Y, Hu H, Su G, Liu H, Shi H, Wu F. Readout-segmented echoplanar diffusion-weighted imaging in the assessment of orbital tumors: comparison with conventional single-shot echo-planar imaging in image quality and diagnostic performance. Acta Radiol. 2017;58:1457-67.

38. Bogner W, Pinker K, Zaric O, Baltzer P, Minarikova L, Porter D, Bago-Horvath Z, Dubsky P, Helbich TH, Trattnig S, et al. Bilateral diffusion-weighted MR imaging of breast tumors with submillimeter resolution using readoutsegmented echo-planar imaging at 7 T. Radiology. 2015;274:74-84.

39. Yeom KW, Holdsworth SJ, Van AT, Iv M, Skare S, Lober RM, Bammer R. Comparison of readout-segmented echo-planar imaging (EPI) and singleshot EPI in clinical application of diffusion-weighted imaging of the pediatric brain. AJR Am J Roentgenol. 2013;200:W437-43.

40. Friedli I, Crowe LA, de Perrot T, Berchtold L, Martin PY, de Seigneux S, Vallee JP. Comparison of readout-segmented and conventional single-shot for echo-planar diffusion-weighted imaging in the assessment of kidney interstitial fibrosis. J Magn Reson Imaging. 2017;46:1631-40.

\section{Publisher's Note}

Springer Nature remains neutral with regard to jurisdictional claims in published maps and institutional affiliations.

Ready to submit your research? Choose BMC and benefit from:

- fast, convenient online submission

- thorough peer review by experienced researchers in your field

- rapid publication on acceptance

- support for research data, including large and complex data types

- gold Open Access which fosters wider collaboration and increased citations

- maximum visibility for your research: over $100 \mathrm{M}$ website views per year

At BMC, research is always in progress.

Learn more biomedcentral.com/submissions 